\title{
Les attentats du 11 septembre. Émotion et raison dans la presse en France et en Italie
}

Emotion and reason in the French and Italian Press after the attacks of

September $11^{\text {th }}$

Emoción y razón en la prensa francesa y italiana despuès de los atentados del 11 de setiembre

\section{Francesca Cabasino}

\section{OpenEdition}

Journals

Édition électronique

URL : https://journals.openedition.org/mots/3003

DOI : $10.4000 /$ mots.3003

ISSN : 1960-6001

Éditeur

ENS Éditions

Édition imprimée

Date de publication : 1 juillet 2004

Pagination : 21-33

ISBN : 2-84788-057-7

ISSN : 0243-6450

\section{Référence électronique}

Francesca Cabasino, « Les attentats du 11 septembre. Émotion et raison dans la presse en France et en Italie», Mots. Les langages du politique [En ligne], 75 | 2004, mis en ligne le 25 mars 2011, consulté le 23 avril 2022. URL : http://journals.openedition.org/mots/3003 ; DOI : https://doi.org/10.4000/mots. 3003 


\section{Les attentats du 11 septembre. Émotion et raison dans la presse en France et en Italie}

Cette analyse de sémio-linguistique comparée porte sur le travail de projections croisées qui a eu lieu dans les médias à la suite de l'inquiétant défi lancé aux États-Unis et à l'humanité par les terroristes du 11 septembre 2001. Des vitrines prestigieuses de la presse nationale, nous tenterons de dégager les modalités d'interaction entre la dimension émotionnelle et les stratégies argumentatives. C'est en effet dans le commentaire que s'actualise un processus de réaction face à un évènement jugé imprévisible et à un nouvel ébranlement des certitudes.

Le corpus choisi est constitué de cinq éditoriaux tirés de trois organes de la presse française et italienne, à deux moments rapprochés mais différents de septembre 2001. Ce critère chronologique permet de suivre l'évolution des points de vue et de vérifier comment s'est effectuée la gestion de la tension entre le pôle de la subjectivité et celui de la représentation collective. Il s'agit des éditoriaux parus dans les livraisons suivantes: Corriere della Sera du 12 septembre, Le Monde et Le Nouvel Observateur du 13; puis Le Monde du 15 et Corriere della Sera du 16.

Notre approche relative à des logiques d'écriture convergentes, où l'aspect formel du message n'est pas sans incidence sur le contenu, s'articulera donc en deux parties: la première essaiera d'appréhender, dans la phase immédiate de l'après-attentat, l'effort consacré à interpréter la symbolique de l'opération terroriste. Les dominantes argumentatives et l'interdiscursivité récurrente dans les réflexions à chaud ne semblent pas résulter d'un acte individuel mais évoquent les sentiments d'une doxa choquée et indignée.

Nous observerons dans le second volet comment la médiation à froid s'engage dans un discours plus complexe, où à l'élan coopératif initial, signe idéalisant qui fait appel à un imaginaire positif contre les forces déstabilisatrices, se substituent des distinguos subtils et des critiques visant la stratégie améri-

1. Université de Rome, La Sapienza - P. le Aldo Moro 5 - francesca.cabasino@uniroma1.it 
caine. Il faudra alors approfondir le rapport ambigu entre les dimensions référentielle et énonciative et les effets produits, dans une perspective communicationnelle, par les stratégies de positionnement du locuteur.

\section{La problématique du soi et de l'autre}

\section{Identifications et délégitimations}

Ce qui frappe dans le dépouillement des journaux des 12 et 13 septembre, c'est la mise en mots d'un état émotionnel indissociable de l'expérience intellectuelle que le locuteur possède du monde. Il n'est pas surprenant que deux directeurs d'importants quotidiens s'appuient sur le même argument pour manifester la solidarité de l'Occident à l'égard des États-Unis et un fort sentiment d'appartenance.

Dans le Corriere della Sera du 12 septembre, le recours à la citation de la phrase célèbre prononcée devant le mur de Berlin, le 26 juin 1963, par John Kennedy manifeste, pour Ferruccio de Bortoli, un engagement explicite de la communauté au niveau planétaire. Certes, le «Ich bin ein Berliner» à la première personne semble n'avoir, d'entrée, que peu de rapport avec le «Nous sommes tous américains » sociopolitique, mais cet appel à la mobilisation n'est ni un simple écho de la source mentionnée ni une recherche de dramatisation à tout prix. La redondance de la formule placée dans l'incipit et au début des trois paragraphes suivants, vise à rechercher un lien à la fois interculturel et politique, construit dans l'espace public un système de valeurs hiérarchisé et fixe dans un acte de langage un principe de cohérence: la nécessité d'une lutte commune $^{2}$. Grâce à ce déclencheur d'émotions, le journaliste tente de transmettre la charge d'inattendu présente dans la nouvelle, en exprimant l'étonnement du groupe social qu'il représente à travers le qualificatif increduli (incrédules) et une profonde inquiétude confiée au participe métaphorique à valeur intensive paralizzati (paralysés).

L'impuissance physique et mentale se répercutant dans le discours raisonné est relevée également par Jean-Marie Colombani dans son long éditorial du Monde daté du 13 septembre, quand il désigne par «le choc que l'on ressent» le sentiment dominant dans la collectivité internationale. Or, c'est pour moduler le stéréotype dans une attitude de distanciation critique que le journaliste fait allusion à la banalité d'un discours de circonstance.

2. En fait, le «Je suis un Berlinois» n'est pas seulement un engagement personnel et moral propre à Kennedy, c'est aussi un message politique, une mise en garde lancée de l'extrême frontière de la guerre froide contre le totalitarisme soviétique. 
Chez de Bortoli, l'implication émotionnelle transparait dans la sobre évocation des victimes de l'attentat, associée à une indication chiffrée approximative et à des groupes nominaux qui mettent en relief la condition du citoyen sans défense: «tante vite ridotte in brandelli e cenere» (de nombreux corps mutilés et réduits en cendres). La condamnation implicite de l'acte terroriste coexiste avec l'allusion à la violation d'un ordre social sécurisant qui entraine de profonds changements: "Anche le nostre vite, più fortunate, cambiano: le ferite che abbiamo dentro sono invisibili ma indelebili» (Même nos propres vies, plus heureuses, en sont changées: les blessures reçues au fond de nous sont invisibles mais indélébiles).

Le thème de la «blessure» intérieure et la présence d'adjectifs négatifs nous font pénétrer dans ce que Charaudeau (2000, p. 141) appelle un «univers de pathémisation ", c'est-à-dire la mise en œuvre socialisée d'un imaginaire émotionnel dans une stratégie d'authenticité, alors que les images strazianti (poignantes) diffusées par les écrans du monde entier pourront difficilement s'effacer de la mémoire. Phrase prémonitoire, parce que de Bortoli, tout en étant conscient de la force visualisante de l'image, n'aurait peut-être pas soupçonné que ces photogrammes de l'horreur reviendraient au ralenti pendant toute l'année 2002 et montreraient encore une fois un pouvoir exceptionnel dû à leur «mutisme pré-sémantique» (Debray, 1994, p. 66).

En revanche, chez le directeur du Monde, malgré le «moment tragique» évoqué à deux reprises, la communication émotive s'oriente, dès le début, vers une forme d'émotion positive. Les principes de proximité et de solidarité ${ }^{3}$ sont réaffirmés à l'aide d'une interro-négative, à fonction assertive, sans marque typographique évidente où l'infinitif d'un verbe psychologique, suivi d'un adverbe évaluatif, sélectionne l'objet du discours: «Comment ne pas se sentir, en effet, comme dans les moments les plus graves de notre histoire, profondément solidaires de ce peuple et de ce pays ». L'inducteur d'émotion coïncide ici avec un système de valeurs où le lecteur peut se reconnaitre et s'engager: c'est le deuxième principe d'inférence émotionnelle théorisé par Ungerer (1995, cité par Plantin, 1998, p. 27).

Dans sa représentation de l'évènement, Colombani utilise une série d'arguments destinés à rejeter lieux communs et généralisations abusives. On peut observer l'appel à un niveau d'adhésion forte dans la réfutation du «déclenchement d'une guerre du Sud contre le Nord». Cette image simplifiée d'une

3. Comme dans l'article italien, la citation de la déclaration de John Kennedy s'intègre au discours du journaliste et fonctionne en argument d'autorité, susceptible de pénétrer «à l'intérieur de l'énoncé» et de valoriser un dire (Mouillaud et Tétu, 1989, p. 140 et 146). Il s'agirait dans ce cas d'un mélange entre la reproduction indirecte et la reproduction polyphonique. 
société planétaire fondée sur l'inégalité, dont seuls les pays riches seraient responsables, vise à disqualifier la position des «belles âmes» prêtes à croire aux «bonnes intentions » d'une opération «qui a frappé tous les symboles de l'Amérique ». La délégitimation / démystification de l'action criminelle, la tentative d'éviter le brouillage, alimenté par la presse en plusieurs occasions, apparaissent dans la suite de l'argumentation, notamment en raison de la présence de deux conditionnels qui soutiennent un raisonnement paradoxal:

Mais dire cela, c'est créditer les auteurs de cette folie meurtrière de «bonnes intentions» ou d'un quelconque projet selon lequel il faudrait venger les peuples opprimés contre leur unique oppresseur, l'Amérique. Ce serait leur permettre de se réclamer de la pauvreté en faisant injure aux pauvres. Quelle monstrueuse hypocrisie!

La stigmatisation d'un langage qui se sert du prétexte de la pauvreté et subvertit un système de valeurs établi vise à augmenter la tension communicative contre un terrorisme de la «bonne cause» et s'exprime par des négations métalinguistiques insérées dans une suite d'énoncés accentuant l'opposition eux / nous: «Aucun de ceux qui ont prêté la main à cette opération ne peut prétendre vouloir le bien de l'humanité. Ceux-là ne veulent pas d'un monde meilleur, plus juste. Ils veulent simplement rayer le nôtre de la carte».

Au contraire des négations "descriptives» destinées à décrire un état du monde, les négations portent ici sur un fragment d'énoncé relatif à la vision mythique du terroriste, dont le locuteur refuse le présupposé.

De toute évidence, le mot terroriste n'est jamais employé; les dénominations sélectionnées, toujours associées à un acte de condamnation, oscillent entre une recherche de précision, comme dans les auteurs de cette folie meurtrière, ces fauteurs de guerre ou des adversaires insaisissables, et une configuration vague, dont témoignent les structures du démonstratif, ceux qui, ceux-là ou du pronom personnel $i l s$, équivalent de la «non-personne». On peut se demander si ces multiples façons de désigner l'autre, mais surtout l'indifférence affichée dans les allusions mentionnées, ne contribuent pas à accentuer la distance qui sépare des individus prêts à tout d'une société agressée.

\section{Les marques discursives de l'émotion}

Dans la seconde partie de l'article du Corriere della Sera, le procédé rhétorique, fondé sur la réitération de la formule choisie pour le titre, favorise la réception du message grâce aux scansions du rythme ternaire et à la relative brièveté des paragraphes. Associé à des structures comparatives pourvues d'une charge émotive remarquable, ce martèlement incessant produit des effets empathiques par la création d'une nouvelle convergence sur les valeurs: 
Siamo tutti americani. Come i passeggieri dei voli dirottati. [...] Come quelle persone che si sporgevano disperate dalle torri [...]. (Nous sommes tous américains. Comme les passagers des vols déroutés. [...] Comme ceux qui se sont jetés des tours par désespoir [...].)

Siamo tutti americani come George W. Bush [...] in una condizione perfino più difficile di quella che dovette affrontare, dopo Pearl Harbor, Roosevelt [...]. (Nous sommes tous américains, comme George W. Bush, dans des conditions encore plus difficiles que celles qu'a dû affronter Roosevelt après Pearl Harbour.)

Siamo tutti americani anche nel guardare con animo affranto e collera crescente le inqualificabili manifestazioni di giubilo palestinese [...]. (Nous sommes aussi tous américains lorsque nous assistons, en spectateurs effondrés puis saisis d'une colère croissante, à l'innommable jubilation des Palestiniens [...].)

Un phénomène formel apte à créer une gradation dramatique contribuerait donc à susciter une prise de conscience, ce que Cosnier (1994, p. 89) appelle «l'organisation du champ sémantique connotatif». Car être citoyen du monde signifie s'identifier à tous ceux qui ont trouvé la mort dans cet attentat, voire, pour de Bortoli, au président américain à l'heure la plus inconfortable de son mandat.

Le dispositif communicatif impliquant émetteur et destinataires construit le passage de la peur à la colère en exploitant l'image dérangeante d'une exultation palestinienne ${ }^{4}$. Si la réalité de ce contre-discours mimo-gestuel diffusé par la télévision, l'hostilité saisie dans des traces vocales incompréhensibles soulignent un plaisir intense né du malheur des autres, l'intention du journaliste est de montrer comment des intérêts divergents parviennent à transformer profondément l'évènement. Il oriente ainsi le regard collectif vers un sentiment d'indignation voué à récupérer un nous provisoirement effacé.

Cette optique n'est pas très éloignée du souci pédagogique de l'éditorialiste du Monde qui, dans la recherche des motivations possibles de l'action terroriste, signale un sentiment négatif, dominé par la violence. C'est la haine que le locuteur constitue en objet langagier marqué, sans l'attribuer à des acteurs particuliers et malgré la présence de procédés d'atténuation destinés à estomper la négativité inhérente au substantif et à relativiser la responsabilité américaine: «Et l'Amérique, dans la solitude de sa puissance, de son hyperpuissance $[\ldots]$ ne semble plus attirer que la haine».

Une argumentation exploitant une relation causale entre deux évènements porte un jugement de valeur sur l'effet: si ce sentiment d'aversion contre les

4. Les doutes exprimés quant à l'authenticité des images des Palestiniens en liesse semblent maintenant dissipés, si l'on en croit les déclarations du journaliste Brusini dans un débat sur les médias audiovisuels (Brusini et al., 2002). 
États-Unis est répandu, c'est à cause de l'absence d'un «contre-modèle soviétique». Or, dans le but de rendre accessibles les véritables enjeux aux lecteurs capables de suivre la logique de ses raisonnements parallèles, Colombani n'exclut pas une remise en cause du comportement peu clairvoyant des Européens. La formule accusatrice sera cependant nuancée et se fondera sur des modalisateurs d'incertitude, tout en focalisant la puissance dangereuse du sentiment: «Et peut-être avons-nous nous-mêmes en Europe [...] sous-estimé l'intensité de la haine qui $[\ldots]$ se concentre contre les États-Unis».

La comparaison avec l'horreur de Pearl Harbour, justifiée par la nature de l'agression et le nombre des victimes, au-delà de l'appel à la compassion, sert à faire évaluer l'évènement criminel et confirme la présence du dispositif argumentatif dans le discours émotionnel. De même, la «haine inextinguible qui nourrit ces attentats » ne possède pas une force illocutoire en soi; ce présupposé explicite devrait faire émerger dans le débat public des conjectures plausibles sur les idéologies destructrices dont s'inspirent les agresseurs ${ }^{5}$ : «Au-delà de leur apparente folie meurtrière, ces derniers obéissent malgré tout à une logique. Il s'agit bien évidemment d'une logique barbare, d'un nouveau nihilisme [...] ».

Si le pathos et l'emphase ne sont pas essentiels dans l'écriture de Colombani, dans la séquence de clôture la peur jamais évoquée apparait sous la forme littéraire du substantif effroi, englobant la crainte impuissante et l'horreur devant une organisation ultramoderne, alors que l'emploi du on collectif, rare tout au long de l'éditorial et suivi ici d'une assertion modalisée, souligne une étrange symétrie avec l'incipit. Afin d'éviter l'ambigüité, le journaliste recourt en outre à la technique de la généralisation dans un énoncé négatif ayant pour objet une folie à l'état pur, sans qualificatifs, qu'aucun désespoir ne peut justifier et dont il refuse une force présumée régénératrice: «Car on peut le dire avec effroi: la technologie moderne leur permet d'aller encore plus loin. La folie, même au prétexte du désespoir, n'est jamais une force qui peut régénérer le monde. Voilà pourquoi, aujourd'hui, nous sommes américains».

Malgré un contexte syntagmatique péjoratif, le retour au nous communautaire et l'évocation de la contrainte temporelle introduisent un élément positif, visent à abolir la distance spatiale et invitent encore une fois à la réflexion collective, à renouer le lien international, à croire dans la dignité et la liberté et à nous sentir différents des terroristes ${ }^{6}$.

5. Pour les dénominations spécifiques de l'attentat, des terroristes et des victimes et pour l'évaluation du discours émotionnel dans la presse, voir l'analyse exhaustive de Koren (1996, chap. 5 et 6 ).

6. Dans la séance exceptionnelle du Sénat du 10 octobre 2001, Lionel Jospin motive ainsi le soutien de la France: «La solidarité qui s'est exprimée à l'égard de nos alliés et amis américains ne tenait pas simplement à l'horreur des actes qui les frappaient, ni aux liens histo- 


\section{Imaginaires discursifs et mise en scène de l'indignation}

Sur la même vague émotionnelle se situe l'article de Jacques Julliard, «L'innommable», dans Le Nouvel Observateur du 13 septembre. Sa condamnation prend déjà place dans ce titre condensé, à valeur d'anathème: toute l'opération est radicalement disqualifiée par un adjectif substantivé. Dans le souci de décrypter ce qu'il définit, en raison de son hypervisibilité, comme un «feu d'artifice terroriste», Julliard estime l'attentat trop ignoble pour le désigner, trop indigne parce qu'il provient d'un adversaire non identifiable et surtout «illisible». Le tragique et la souffrance, les «blessures matérielles et symboliques infligées à la première puissance mondiale» au nom d'une vague idéologie «nous coupent le souffle», comme si, dans cette tentative d'identification/projection destinée au public, l'indignation, mêlée à l'étonnement, était la seule réaction possible devant un irrationnel grandissant, une escalade dans la barbarie, devant «l'absurdité totale» à laquelle nous sommes confrontés.

La tension émotive se reconnait dès le début dans la perception sonore, le «cri» déchirant qui secoue la planète face aux «incroyables nouvelles» venues des États-Unis: «C'est la guerre». Une tension neutralisée cependant dans l'accumulation laconique de détails réalistes sur l'effondrement des tours (en cendres) ou sur le sort du Pentagone (en flammes) et vite réveillée par le procédé syntaxique de l'interrogation juxtaposée. Des énoncés minimaux au rythme haletant («Venues d'où? De Russie? De Chine? Du Moyen-Orient?») mettent en évidence l'effort d'avancer des conjectures, sans pouvoir les confirmer, sur la provenance des attaques. L'émotion réapparait enfin dans l'évocation de termes canoniques comme tragédie, horreur, horrible, effarant, barbarie, terreur, terribles attentats, degré d'atrocité, appartenant à l'aire sémantique de la peur.

En dépit d'une discontinuité de surface et d'un ordre d'apparition des arguments plutôt incertain, le cadrage que le locuteur construit tourne autour d'un univers de référence qu'il souhaite commun: «Et pourtant il faut résister à l'emploi du mot guerre». Or, ce refus de la désignation coexiste avec la récurrence d'un terme attesté onze fois sous différentes formes lexicales. Un mot remis en question se trouve donc au cœur du régime énonciatif du journaliste qui observe «l'entrée dans un monde nouveau» par une transformation profonde des «conflits classiques» et de «leurs rituels». Il suffit de relire l'avant-dernier paragraphe, où convergent la plupart des occurrences du nom suivies d'un adjectif ou de la construction $d e+$ substantif, pour saisir le contraste entre une situation de stabilité relative connue pendant la guerre froide (guerre entre puissances) et

riques qui nous liaient à ce pays; elle tenait également au sentiment que nous étions, nous aussi, potentiellement menacés par le terrorisme et que cette lutte qu'il fallait entreprendre était également la nôtre». 
l'absence actuelle de perspectives pour une paix durable: guerre invisible, guerre de tous contre tous, guerre de tous les instants, guerre permanente des hommes contre eux-mêmes.

Ces assertions sont en mesure de montrer la réalité d'une menace permanente, conséquence d'un «terrorisme exercé à une échelle sans précédent». Une nouvelle «doctrine de dissuasion du faible au fort» est à l'œuvre, qui met en relief, à cause du renversement des positions stratégiques, la fragilité de l'Occident et son incapacité de «répondre du tac au tac». Or, la concrétisation de cette vulnérabilité, qui produit un conflit asymétrique, permet à Julliard de faire émerger la remise en cause des stéréotypes sur la relation complexe faible/fort et d'évoquer implicitement le problème de l'insécurité planétaire.

Au lieu d'admettre son impuissance à guider un public désorienté, le journaliste se focalise sur la description d'une réalité jugée inacceptable. En effet, son discours axiologique contre une violence aveugle incapable d'assurer l'un des principes démocratiques revendiqués, l'idéal de justice, recourt dans la phrase finale à la métaphore des monstres qui se réveillent. On peut observer dans ce transfert analogique un exemple de rhétorique «tensive» (Bertrand, 2000, p. 30), fonctionnant comme un opérateur d'émotion. L'intention de Julliard, à travers une remotivation figurale fondée sur l'hyperbole, est d'engager un débat sur la négation des valeurs fondamentales que le terrorisme représente, malgré une prétention moralisante. À cet égard, les forces brutales agissant contre la civilisation servent à l'auteur pour imposer une relation antiphrastique et établir un conflit conceptuel in absentia avec tout projet rationnel. En même temps, le jeu de l'analogie conduit à créer, au niveau intratextuel, des connexions entre la monstruosité relevée et l'absurdité ou la barbarie qui risquent de contaminer l'Occident. Privilégiés dans des endroits cruciaux, ces mots accentuent l'objectif de mise en garde et le degré d'adhésion de l'énonciateur.

\section{Stratégies de distanciation}

Quelques jours après l'effondrement des tours de Manhattan, le positionnement à implication émotionnelle subit une évolution. En effet, le devoir de responsabilité à l'égard du public comporte aussi un dépassement du comportement défensif; les pulsions dictées par l'urgence d'une condamnation cèdent la place à l'analyse raisonnée des problèmes; le logos parait l'emporter sur le pathos. Aux argumentations disqualifiant l'attaque, à la rhétorique du lien participatif et au langage de l'inquiétude qui avaient caractérisé la chambre d'écho médiatique se substituent des réflexions de nature morale sur les faits. 


\section{Contre les interprétations dualistes de l'évènement}

L'éditorial non signé du Monde du 15 septembre, au titre injonctif «Refuser le manichéisme», représente une ligne de fracture par rapport aux propos antérieurs. Il s'ouvre sur un énoncé qui semble remettre en cause certains des présupposés initialement activés par Colombani:

L'émotion ressentie à travers le monde après les attentats de New York et de Washington, la solidarité naturelle et spontanée manifestée, notamment en Europe, avec le peuple américain et ses dirigeants ne justifient pas des conclusions simplistes.

L'émotion est encore au cœur des représentations collectives, mais son impact parait moins englobant, alors que la recherche de la vérité et le refus de la fausse dialectique du bien et du mal deviennent l'objectif prioritaire du quotidien: «Mais cette sympathie profonde ne nous oblige pas à tomber dans le piège du manichéisme cher aux sectes protestantes qui ont fait l'Amérique».

L'attitude fortement réfutative est perceptible dans le «non» réitéré, souligné par la rigidité du rythme binaire, qui introduit les deux arguments-clés de l'article:

Non, la lutte nécessaire contre le terrorisme international n'est pas un combat monumental entre le Bien et le Mal, contrairement à ce qu'a déclaré George W. Bush. Non, l'attaque contre les centres vitaux et symboliques du capitalisme triomphant par des kamikazes venus de pays musulmans n'a pas été engendrée par un clash des civilisations, selon l'expression du professeur Samuel Huntington.

Contre une «tentation intellectuellement erronée et politiquement dangereuse » qui rappelle un populisme de matrice autoritaire ou qui fait des conflits identitaires le motif principal des actions terroristes, le recours explicite à la rigueur rationnelle est une étape obligée: «Si la raison a encore un sens dans les sociétés modernes et développées, c'est précisément parce qu'elle s'oppose au fanatisme, aux amalgames, aux condamnations précipitées et collectives ».

Le nouvel engagement de la rédaction du Monde évoque implicitement la fin de l'état d'urgence, s'oppose à la diabolisation des musulmans et nuance les modalités du soutien aux États-Unis en fixant les conditions du dialogue, seulement «s'ils acceptent de coopérer, de discuter et de partager avec les autres pays dans une lutte de longue haleine contre la terreur». Il semble dicté par la prise de conscience que le désir d'identification commence à s'estomper et qu'il faut réinventer la solidarité sans se laisser capturer par les accents de croisade du président américain: «C'est d'une telle politique déterminée mais réfléchie que nous pouvons nous sentir solidaires, pas de la dialectique de Dieu et du diable». 


\section{Des sentiments globalisés à la réfutation de l'émotion}

Il est intéressant d'observer comment un éditorial bref, mais incisif et plein de suggestions, ne tardera pas à enrichir le débat dans la presse italienne. Ainsi l'ancien ambassadeur Sergio Romano dans «Emozioni e ragione» (émotions et raison), Corriere della Sera du 16 septembre 2001, n’hésite pas à créer des liens intertextuels avec Le Monde à travers le dispositif de l'allusion, tout en proposant son analyse approfondie des évènements. Après avoir rappelé le deuil partagé par la planète comme une forme de globalisation, consécutive à des comportements réactionnels homologues, il construit un espace discursif dominé par la rationalité, en utilisant avec précaution un argument d'autorité: "occorre tornare, il più rapidamente possibile, alla normalità» (revenir le plus rapidement possible à la normale). Sa prise de position, vouée à démasquer les intentions de l'action terroriste, ne se veut pas polémique; elle entend marquer une dissociation par rapport aux interprétations courantes:

I terroristi non vogliono soltanto uccidere e distruggere. Vogliono soprattutto sconvolgere la nostra vita, sovvertire le nostre consuetudini, obbligarci a sospendere le nostre garanzie democratiche [...]. Vogliono dirottare la nostra vita quotidiana. (Les terroristes ne veulent pas seulement tuer et détruire. Ils veulent par dessus tout bouleverser notre façon de vivre, subvertir nos habitudes, nous obliger à laisser lettre morte nos garanties démocratiques [...]. Ils veulent dérouter notre vie quotidienne.)

Jouant sur l'antiphrase et la juxtaposition de structures parallèles, Romano confirme cette volonté de destruction, mais lance aussi un appel explicite à la convergence et se sert de l'emploi figuré du verbe dérouter, mis en valeur (les avions le furent au 11 septembre), pour rendre tangible son interprétation du réel. Il réalise ainsi ce dédoublement dans l'interprétation des configurations linguistiques dont Searle (1982, p. 131 et suiv.) avait pressenti l'importance et qui impose un sens concret plus fort que le sens habituel du mot.

Dans la suite de l'argumentation, il souligne que les émotions, aussi légitimes qu'elles soient, ne doivent pas se traduire en décisions politiques précipitées. En reliant les droits et les devoirs au principe des «garanties démocratiques», il s'appuie sur le paradigme de la légitimité/acceptabilité. L'énonciation se concentre d'abord sur la formule «ha diritto di» (a le droit de), référée alternativement aux États-Unis, désireux de «punir les responsables», à Israël qui veut "protéger ses concitoyens » et à l'Occident démocratique obligé de «se défendre contre le fanatisme islamique». Répétée au début de trois énoncés consécutifs, tout en réaffirmant des valeurs fondamentales, l'expression ouvre la voie à une remise en question et laisse entrevoir une impossibilité de répondre à l'exigence de justice. Une stratégie réfutative est donc à l'œuvre, confiée au mais d'opposition ou à des structures déontiques négatives comme: 
«L'Occidente non deve attribuire all'Islam gli errori delle sue sette più radicali» (L'Occident ne doit pas attribuer à l'Islam les fautes de ses sectes les plus radicales).

Revenant sur le thème de la solidarité, Romano inverse l'équation. L'objet de réflexion est devenu le devoir des pays européens d'assurer toute la collaboration possible avec les Américains, même si l'insinuation du doute sur l'application de l'article 5 du traité de l'OTAN entend rétablir le processus rationnel qui devrait précéder toute prise de décision.

Dans le dernier paragraphe, la reprise des paroles initiales, « revenir à la normale », vise à préciser un message qui prétend raviver le débat sur la substance du terrorisme et la recherche des raisons qui ont permis à ses leaders d'exploiter, au Proche-Orient et en Asie, le ressort passionnel des populations. Mais c'est surtout dans la conclusion que la dissociation entre émotion et rationalité atteint son sommet. On est à nouveau confronté à une double polarisation introduite par l'indéfini négatif nessuno:

Nessuno, nei prossimi anni, potrà ricordare gli attentati di New York e Washington senza provare rabbia e indignazione. Ma nessuno, speriamo, dimenticherà che la migliore politica ha sempre il sangue freddo e sa mantenere la testa sulle spalle. (Personne, dans les années qui viennent, ne pourra se souvenir des attentats de New York et de Washington sans éprouver de la rage et de l'indignation. Mais personne, nous l'espérons, n'oubliera que la meilleure politique réside toujours dans le fait de garder son sang-froid et la tête sur les épaules.)

Si le souvenir de cet acte d'intimidation est susceptible de justifier et de remotiver l'émotion, la quête du rationnel reste, pour les dirigeants politiques et les journalistes, le seul point d'ancrage apte à refonder les valeurs affaiblies des sociétés démocratiques ${ }^{7}$.

Au-delà de l'évidente complémentarité des textes argumentatifs analysés, la mise en discours de l'émotion a montré d'abord comment se construisent les images mentales de l'autre, défini négativement comme un ennemi ou passé relativement sous silence, et du même, fragilisé parce que croyances et sym-

7. Dans l'optique de la convergence/opposition entre émotionnel et rationnel, il n'est pas sans intérêt de mentionner l'opinion défendue dans l'article d'Umberto Eco «Le guerre sante: passione e ragione» (La Repubblica, 5 octobre 2001). Selon le sémiologue italien, «toutes les guerres de religion sont nées d'adhésions passionnelles à des oppositions simplistes: Nous et les Autres, bons et méchants, Blancs et Noirs. Si la culture occidentale s'est montrée féconde, c'est aussi parce qu'elle s'est efforcée de "dissoudre" les simplifications à la lumière de la recherche et de l'esprit critique et parce qu'elle a élaboré la capacité de mettre à nu ses propres contradictions». 
boles se sont soudain évanouis, mais capable de réagir et de s'engager dans des actions solidaires. Elle a permis ensuite de souligner la double fonction, délégitimante et justificatrice, de la désignation dans un contexte d'éditoriaux qui ne peuvent éviter de dire l'innommable. Dans la tentative de clarifier les ambiguïtés et d'envisager causes et conséquences de l'attaque, les professionnels de la presse écrite cherchent à s'adapter à la capacité critique du lecteur impliqué. C'est ainsi qu'ils laissent découvrir la séduction qu'un état affectif comme la haine exerce sur les acteurs du crime, qu'ils invitent à refuser l'amalgame pauvreté/violence ou parviennent à rendre perceptibles sous des représentations métaphoriques les sentiments de crainte et d'indignation. Ils actualisent donc une rationalité de type subjectif qui crée des effets empathiques puisque fondée sur des valeurs partagées.

Alors que, dans une première phase, se dégage une prééminence de l'intentionalité pathémique associée à un état d'angoisse entrainant progressivement des émotions négatives, c'est surtout le sentiment de vulnérabilité, reflet de l'imaginaire occidental, qui conduit à la prise de conscience d'une marque identitaire profonde et à la réaffirmation unanime des principes démocratiques. Lié au drame et aux réactions suscitées, cet effort d'identification assume une force illocutoire indéniable et encourage le récepteur à évaluer l'opération terroriste selon son propre système émotionnel.

Dans la seconde phase, on observe une transformation des modalités d'écriture oscillant entre des stratégies de réfutation de l'émotion et la nécessité de repenser la solidarité selon de nouveaux paramètres, en conciliant le respect de la diversité avec le besoin de communauté. Mais, pour atteindre cet objectif, il aura fallu que la dimension émotionnelle participe de la rhétorique de l'indicible, car, si l'on en croit Braud (1996, p. 8), «les affects se situent aussi au cœur des processus de construction des intérêts, des aspirations et des exigences ».

\section{Bibliographie}

BERTRAND D., 2000, «Enthymème et textualisation », Langages, n 137, mars, p. 29-45.

BRAUD P., 1996, L'émotion en politique, Paris, Presses de Sciences Po.

BRUSINI H. et al., 2002, «11 septembre 2001. La guerre en direct», MédiaMorphoses, $\mathrm{n}^{\circ} 4$, mars, p. 4-16.

CHARAUDEAU P., 2000, «Une problématisation discursive de l'émotion. À propos des effets de pathémisation à la télévision », C. Plantin, M. Doury et V. Traverso (dir.), Les émotions dans les interactions, Lyon, PUL, p. 125-155.

COSNIER J., 1994, Psychologie des émotions et des sentiments, Paris, Retz-Nathan.

DEBRAY R., 1994, «Vie et mort de l'image. Une histoire du regard en Occident», Esprit, $\mathrm{n}^{\circ} 199$, février, p. 57-66. 
KOREN R., 1996, Les enjeux éthiques de l'écriture de presse et la mise en mots du terrorisme, Paris, L'Harmattan.

MOUILLAUD M. et TÉTU J.-F., 1989, Le journal quotidien, Lyon, PUL.

PLANTIN C., 1998, «Les raisons des émotions», M. Bondi (dir.), Forms of Argumentative Discourse, Bologne, CLUEB, p. 3-50.

SEARLE J., 1982, Sens et expression, Paris, Minuit.

\section{Résumé / Abstract / Compendio}

\section{Les attentats du 11 septembre. Émotion et raison dans la presse en France et en Italie}

L'analyse de la mise en discours de l'émotion dans les éditoriaux à la suite de l'opération terroriste du 11 septembre permet de dégager deux attitudes. Dans la phase immédiate de l'après-attentat, un sentiment de vulnérabilité engendre la prise de conscience d'une profonde appartenance, alors que la distance du drame impose un appel à la rationalité pour refonder la solidarité sur les valeurs démocratiques.

Mots-clés : discours politique, émotion, éditoriaux, opération terroriste, 11 septembre.

\section{Emotion and reason in the French and Italian Press after the attacks of September $11^{\text {th }}$}

The present analysis deals with emotion in discourse with a particular reference to the editorials that were published after the terrorist attacks against the United States. There were two types of reactions in the Press: immediately following the attacks, an impression of vulnerability conjured up a sense of identity and solidarity. After this first emotional reaction, there was an appeal to rationality in which solidarity is based on democratic values.

Keywords: political discourse, emotion, editorials, terrorist attacks, 9 / 11.

Emoción y razón en la prensa francesa y italiana despuès de los atentados del 11 de setiembre

El análisis de la puesta en discurso de la emoción en los editoriales consecutivos al 11 de setiembre permite destacar dos atitudes. En una primera fase siguiendo el ataque se desarrolló un sentimiento de vulnerabiliad desencadendando pues una toma de conciencia de una pertenencia cuando la distancia del drama imponía un llamado a la conciencia para fundar de nuevo la solidaridad sobre valores democráticos.

Palabras claves : discurso politico, emoción, editoriales, ataque terrorista, 11 de setiembre. 